\title{
An experimental administration of pravastatin in patient with previous, multiple pregnancy losses
}

\author{
Szymon Kozłowski, Michał Lipa, Jacek Lipa, Dorota Bomba-Opoń \\ $1^{\text {st }}$ Department of Obstetrics and Gynecology of Medical University of Warsaw, Poland
}

A 33-years old patient gravida 5 with chronic hypertension, hypothyroidism, polycystic ovarian syndrome with insuline resistance and obesity (BMI 32) was offered an experimental administration of pravastatin due to multiple pregnancy losses (fetal demise at $6,8,18$ and 22 weeks) and preeclampsia in the last pregnancy. From the early first trimester methyldopa ( $750 \mathrm{mg} /$ day), metformin ( $850 \mathrm{mg} /$ day), L-thyroxine $(50 \mu \mathrm{g} /$ day $)$, acetylsalicylic acid ( $150 \mathrm{mg} /$ day) and enoxaparin $(40 \mu \mathrm{g} /$ day) were administered. First trimester risk assessment after combined test revealed low risk of chromosomal abnormalities with high risk of preeclampsia and fetal growth restriction: pregnancy associated plasma protein A (PAPP-A) serum level corresponding to 0.294 multiple of median (MoM) and high pulsatility indices in uterine arteries. At 17 weeks of gestation pravastatin ( $20 \mathrm{mg} /$ day) was included to the treatment as a preventive modality from preeclampsia and intrauterine fetal demise (IUFD). Before administration of the pravastatin, patient gave written consent and this management was accepted by Ethical Comittee of Medical University of Warsaw. Anomaly scan did not reveal any abnormalities. At 27 weeks of gestation patient was admitted to the hospital due to cervical insufficiency with estimated fetal weight corresponding to $12^{\text {th }}$ centile, normal volume of amniotic fluid and cerebroplacental ratio (CPR). Due to high risk of preterm delivery, cervical pessary and course of $24 \mathrm{mg}$ betamethasone i.m. were administered. Throughout prental surveillance we observed fetal growth restriction (FGR) with decreasing volume of amniotic fluid, brain sparing and decreasing short time variability (STV) in cardiotocography (Figure 1). At 33 weeks of gestation, due to high risk of intrauterine fetal demise, the caesarean section was performed. A female newborn was delivered in good general condition (10 points in Apgar score) with birthweight 1545 grams. Postnatal placental examination revealed morphologically normal placenta

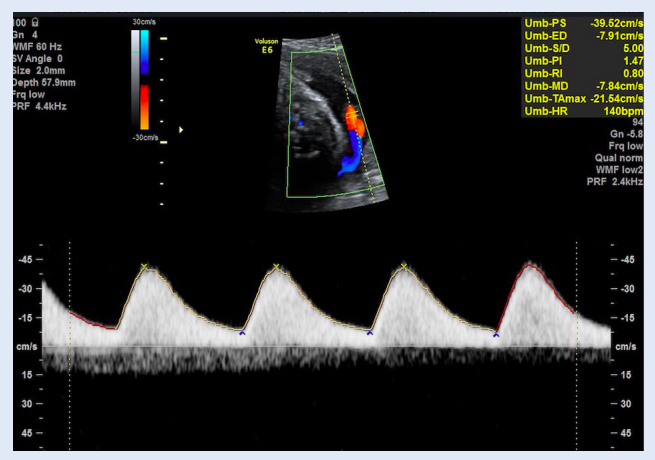

Figure 1. Increased pulsatility index (PI) $\left(>95^{\text {th }}\right.$ centile) in umbilical artery at 33 weeks of gestation

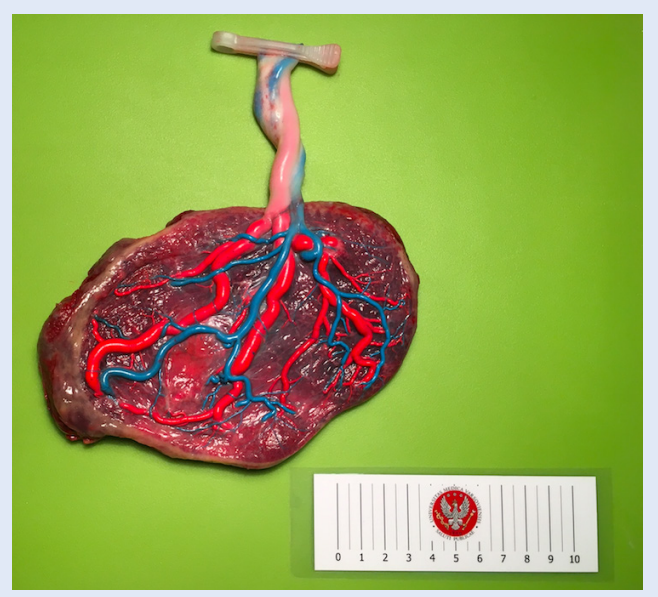

Figure 2. Postnatal, dye injection examination revealed three vessels in umbilical cord and marginal cord insertion. Placental mass weight 245 grams corresponding to $10^{\text {th }}$ centile with weight 245 grams (corresponding to 10th centile at this gestational age), three vessels umbilical cord and marginal cord insertion (Figure 2). Subsequently, newborn was transferred to Neonatal Intensive Care Unit (NICU). Due to transient respiratory adaptation problems a nasal continuonus positive airways pressure (nCPAP) was administered for 5 days. During the stay in NICU an anemia and increased thyroid stymulating hormone (TSH) concentration were detected. After 
28 days, the neonate was discharged from Neonatal Department with body weight 2040 grams. Currently, the infant develops normally and remains under care of Pediatric Out Patient Department.

To our knowledge, this is the first case of pravastatin admnistration in Poland in high risk pregnant woman. Due to side effects, statins are not routinely administered in pregnancy, however, such an experimental approach may be considered in patients with recurrent, early onset preeclampsia resulting in pregnancy failure. Beneficial impact of the pravastatin may prevail the side effects in specific group of patients, nonetheless more studies need to be undertaken to include this management in routine clinical practice. 